Itinéraires Itinéraires

Littérature, textes, cultures

2020-2 | 2020

Discours animal. Langages, interactions, représentations

\title{
La linguistique et le langage animal. Résistances, décentrements, propositions
}

Linguistics and Animal Language. Resistances, Decentrings, Proposals

Marie-Anne Paveau et Catherine Ruchon

\section{CpenEdition}

Journals

Édition électronique

URL : https://journals.openedition.org/itineraires/8202

DOI : $10.4000 /$ itineraires.8202

ISSN : 2427-920X

Éditeur

Pléiade

Référence électronique

Marie-Anne Paveau et Catherine Ruchon, «La linguistique et le langage animal. Résistances,

décentrements, propositions », Itinéraires [En ligne], 2020-2 | 2020, mis en ligne le 22 décembre 2020, consulté le 24 novembre 2021. URL : http://journals.openedition.org/itineraires/8202 ; DOI : https:// doi.org/10.4000/itineraires.8202

Ce document a été généré automatiquement le 24 novembre 2021.

\section{(†) $\odot$

Itinéraires est mis à disposition selon les termes de la licence Creative Commons Attribution - Pas d'Utilisation Commerciale - Pas de Modification 4.0 International. 


\title{
La linguistique et le langage animal. Résistances, décentrements, propositions
}

\author{
Linguistics and Animal Language. Resistances, Decentrings, Proposals
}

\author{
Marie-Anne Paveau et Catherine Ruchon
}

1 Alors que de nombreuses disciplines se sont intéressées de longue date ou s'intéressent désormais à la production de significations par les animaux, les sciences du langage semblent résister et camper sur des positions anthropocentrées les isolant de plus en plus d'un large mouvement contemporain qui se déploie dans les sciences humaines et sociales au niveau international. Se développe en effet, sur l'impulsion des travaux précurseurs de Latour (1991), Descola (2006) ou Derrida (2006) notamment (pour le champ francophone), une conception écologique du monde appuyée sur une remise en cause des grandes distinctions binaires qui fondent la pensée dite occidentale, et, au premier chef, la distinction entre humain et animal. Des sciences humaines et sociales au-delà de l'humain et avec le non-humain sont en train d'apparaitre. Ce mouvement accompagne une évolution politique mondiale sur le statut des animaux : militante's et politiques, à des degrés divers selon les zones géographiques et les conditions socioéconomiques, accordent de plus en plus aux animaux une place véritable parmi les êtres vivants.

2 Dans cet article, nous voudrions essayer de comprendre ce qui bloque les linguistes dans une humanité (leur humanité ?) qui se fait parfois anthropocentrisme étroit, coupé de l'animalité, et d'identifier les verrous qui les empêchent, alors même que leurs collègues éthologues, philosophes, cognitivistes, anthropologues, sociologues ou psychologues posent largement la question du langage animal, de prendre en compte des éléments de recherche hors de "l'exception humaine" (Schaeffer 2007). Par linguistes, désignation que nous savons trop généraliste et stéréotypée, nous entendons l'ensemble des chercheure's qui travaillent dans les frontières mainstream de la discipline, et qui contribuent à son maintien en tant que telle, tant sur le plan national qu'international. 
3 Nous décrirons d'abord la doxa culturelle et professionnelle des linguistes et plus généralement des sciences humaines et sociales, nourrie de références cultivées sur la spécificité exclusivement humaine du langage, assurant la prégnance d'une conception axiologique négative de l'anthropomorphisme, fondée sur ce que Frans De Waal appelle l'anthropodéni (de Waal 2001). Nous montrerons ensuite qu'à contrario, la question animale est entrée, parfois largement, dans les préoccupations de bien des disciplines tant du côté des sciences de la nature que des sciences humaines et sociales, et souvent par le biais de la communication et du langage. Nous examinerons ensuite plus précisément la construction scientifique de la résistance de la linguistique à l'intégration des données animales, résistance fondée en grande partie sur les notions d'articulation du langage et de symbolisation, dont la célèbre discussion entre Benveniste et von Frisch sur la danse des abeilles est emblématique (Benveniste 1952). Nous proposerons enfin quelques pistes et orientations méthodologiques pour travailler le langage animal et l'intégrer aux questionnements des sciences du langage.

\section{Doxas : anthropodéni et anthropomorphisme}

«L'homme est la mesure de toute chose » : c'est sans doute la doxa universellement prévalente jusqu'à nos jours, que ses racines millénaires et son apparente évidence rendent difficile à contester. En fait, cette si célèbre phrase, prononcée par Protagoras dans le Théétète, et non par Platon comme on le croit souvent, ne veut pas dire ce que l'on croit : c'est une déclaration d'empirisme, de subjectivisme et de mobilisme qui vise à contester la possibilité de la science; c'est l'énoncé fondateur du relativisme. Contre cette conception, Platon défend, on le sait, la possibilité de la science grâce aux vérités mathématiques et à l'existence d'une réalité intelligible (Platon, Théétète). Mais il n'empêche que cette déclaration est devenue dans le fonds commun un emblème de la suprématie humaine.

\subsection{Culture chrétienne et Antiquité}

5 L'anthropocentrisme de la culture contemporaine occidentale se fonde plutôt sur le christianisme, considéré par l'historien Lynn Townsend white comme la religion la plus anthropocentrique du monde (White 1967 : 1205). Cette idée est développée par la philosophe Virginie Maris (2016) pour qui « la culture judéo-chrétienne place l'homme au sommet de la création ». La Genèse, le premier livre de la Bible, reflète le désir de domination de l'humain sur les animaux :

Dieu dit faisons un adam à notre image comme notre ressemblance. Pour commander au poisson de la mer, à l'oiseau du ciel, aux bêtes et à toute la terre, à toutes les petites bêtes ras du sol. (I, 26)

Dieu crée l'adam à son image, le crée à l'image de Dieu, les crée mâle et femelle. Dieu les bénit et leur dit (I, 27).

À vous d'être féconds et multiples, de remplir la terre, de conquérir la terre, de commander au poisson de la mer, à l'oiseau du ciel, à toutes les petites bêtes ras du sol. (I, 28) (Genèse 1, 26-28, La Bible. Nouvelle traduction, Bayard)

6 Le philosophe allemand Arthur Schopenhauer avait déjà établi en 1871 le lien entre le comportement des humaine's envers les animaux et le mythe de la création dans l'Ancien Testament que reprend le christianisme (Schopenhauer [1851] 2005 : § 177). Il critique violemment la morale chrétienne, qui n'a «nul égard pour les bêtes » et qui a 
conduit les humaine's à traiter les animaux comme des choses (Schopenhauer [1839] 1879 : 194).

7 Les philosophes antiques ont aussi transmis l'idée de la suprématie humaine par cette fameuse maxime réinterprétée, "L'homme est la mesure de toute chose» de Protagoras, et par la conception aristotélicienne des animaux comme des êtres sentants (par opposition aux objets sentis, c'est-à-dire passifs) mais dépourvus du logos et de toute forme d'intellect: "et l'homme, seul de tous les animaux, possède la parole " (Aristote, La Politique, 1962 : 26). Le disciple de Platon a consacré de nombreux traités au monde animal au $\mathrm{IV}^{\mathrm{e}}$ siècle av. J.-C., basés sur une observation rigoureuse des animaux mis à sa disposition par Alexandre. Son système taxinomique, notamment dans le livre IV de son Histoire des animaux, écrit vers 343 av. J.-C., a fourni le socle d'une classification hiérarchisée (scala naturae) qui a cours aujourd'hui encore, depuis les êtres inanimés jusqu'aux plantes (inférieures et supérieures), aux mollusques, aux quadrupèdes vivipares et aux êtres humains (De Wit et Cornelius 1994 : 5-10).

Figure 1. Dessin de la scala naturæ par Didacus Valades, Rhetorica Christiana (1579), domaine public.

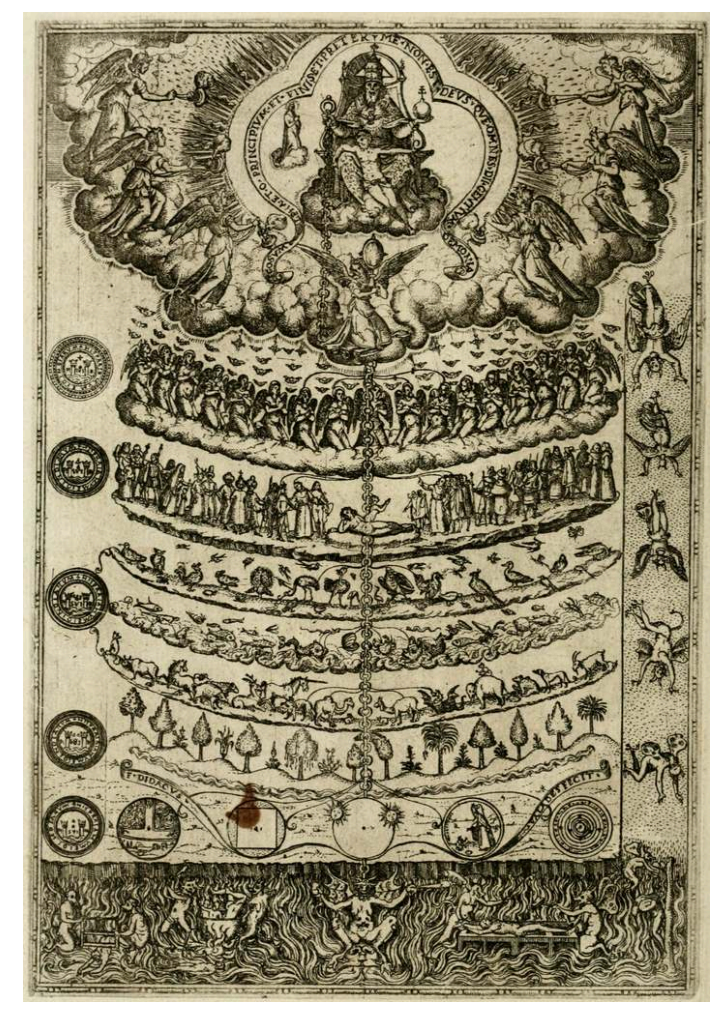

La classification hiérarchisée des animaux d'Aristote perdure jusqu'au XVIII ${ }^{\mathrm{e}}$ siècle et Buffon s'en inspire pour son Histoire naturelle, une encyclopédie en trente-six volumes (1749-1789) au grand succès.

8 Cependant Aristote, responsable malgré lui de cette hiérarchisation nécessaire à son classement, ne contestait pas la sensibilité de l'animal qu'il étudie de façon très pointilleuse dans son quatrième livre, au chapitre VIII, « Histoire des animaux » : « Le sens du toucher appartient à tous les animaux» (Aristote, Histoire des animaux, 1883 : $\S 20$ ). Et plus loin : «Tous les animaux pourvus d'une bouche jouissent, ou souffrent, de la saveur des objets qu'ils touchent. Quant à la vue et à l'ouie, les observations ne sont, 
ni bien certaines, ni bien évidentes " (Aristote, Histoire des animaux, 1883 : § 25). Autre fait notable chez Aristote : c'est l'un des naturalistes qui a le mieux étudié la question de la voix chez les animaux. Pour exemple, à propos du dauphin et des oiseaux, dans le chapitre IX, intitulé « De la voix des animaux » :

Le dauphin a bien une voix, puisqu'il a un poumon et une trachée-artère ; mais il n'a pas la langue libre, et il n'a pas de lèvres, de manière à pouvoir articuler quelque chose avec cette voix. (Aristote, Histoire des animaux, 1883 : § 9)

Les oiseaux émettent une voix; et ceux-là même l'articulent le mieux qui ont une langue assez large, ou qui ont la langue très mince. Dans quelques espèces, la voix du mâle et celle de la femelle sont tout à fait pareilles; dans d'autres, elles sont différentes. (Aristote, Histoire des animaux, 1883 : § 13)

Certes, l'unité de mesure reste l'humain qui garde le privilège du langage :

Les quadrupèdes vivipares ont chacun des voix différentes les unes des autres; mais aucun n'articule un langage; ce privilège est réservé à l'homme seul. Quand l'animal a un langage articulé, il a aussi une voix; mais il peut avoir une voix sans toujours avoir de langage, ni d'articulation. (Aristote, Histoire des animaux, 1883 : $\S 15)$

\subsection{Le travail spéciste de Descartes}

9 La résistance spéciste contre l'idée d'un langage animal prend sa source au XVII ${ }^{e}$ siècle dans le Discours de la méthode de Descartes et sa thèse de l'animal-machine qui présente les animaux comme des automates dénués de raison et répondant à des stimuli (Descartes 1637). Nicolas Malebranche, philosophe et théologien français, prolongera dans ses écrits la conception de Descartes :

Dans les animaux il n'y a ni intelligence, ni âme, comme on l'entend ordinairement. Ils mangent sans plaisir, ils crient sans douleur, ils croissent sans le savoir : ils ne désirent rien, ils ne craignent rien, ils ne connaissent rien : et s'ils agissent d'une manière qui marque intelligence, c'est que Dieu les ayant faits pour les conserver, il a formé leur corps de telle façon qu'ils évitent machinalement et sans crainte tout ce qui est capable de les détruire. Autrement il faudrait dire qu'il y a plus d'intelligence dans le plus petit des animaux, ou même dans une seule graine que dans le plus spirituel des hommes : car il est constant qu'il y a plus de différentes parties et qu'il s'y produit plus de mouvements réglés que nous ne sommes capables d'en connaître. (Malebranche $1678: 432$ )

Descartes avait comparé l'animal à une horloge, tout en lui accordant une certaine émotivité. Malebranche va plus loin et dénie toute sensibilité à l'animal. Dans leur étude sur l'évolution épistémologique du concept d'animal au regard de la dialectique sujet/objet, Michel Vidal et Laurence Simonneaux (2014) rapportent cette anecdote signifiante : après avoir donné un coup de pied à un chien, Malebranche aurait affirmé : «Eh quoi, ne savez-vous pas que cela ne sent point?»

Descartes utilise l'absence du langage comme argument pour démontrer que l'animal n'est pas un sujet, c'est-à-dire un être doté de pensée (Vidal et Simonneaux 2014). Il leur réfute aussi toute forme de langage intraspécifique : «Et on ne peut dire qu'elles parlent entre elles mais que nous ne les entendons pas; car, comme les chiens et quelques autres animaux nous expriment leurs passions, ils nous exprimeraient aussi bien leurs pensées, s'ils en avaient " (Descartes, cité dans Vidal et Simonneaux 2014 : $\S 6)$.

11 Le philosophe admet une physicalité commune à l'humain et à l'animal, mais place la pensée hors du corps matériel. La conception de Descartes marque durablement les 
sciences de la nature et les sciences dites «humaines». Au point qu'un scientifique comme l'anthropologue Jean-Pierre Digard défend l'idée que l'animalisme est un antihumanisme (Digard 2018) et conteste l'idée que la culture ne soit plus le propre de l'homme, ce qui « abaisse l'homme au rang d'“animal humain" " (Digard 2000).

Ces éléments constituent un verrou idéologique potentiellement commun à l'ensemble des chercheures's, qu'il s'agisse des sciences humaines ou sociales, des sciences physiques ou des sciences de la nature, que l'on appellera, selon ses manifestations, anthropocentrisme ou supériorité humaine, ce verrou étant par ailleurs profondément lié à d'autres verrous analogiques comme le phallocentrisme et l'occidentalocentrisme. En dépit de farouches opposant-e-s à l'idée d'une pensée animale et encore plus à un langage animal, l'histoire est jalonnée de penseur·es qui ont épousé la cause animale bien avant que ne s'expriment les tendances animalistes actuelles.

\section{La prise en compte contemporaine de l'animal}

La question animale s'est développée dans l'espace public mais également dans la recherche en sciences humaines et sociales, questionnant la doxa spéciste sur laquelle reposent bien des fondements de nos sociétés. Dans les trente dernières années, la prise en compte de l'animal a progressé pas à pas, devenant plus visible ces dix dernières années, puisque militant·e's, intellectuelle's et chercheur'e's se sont mis'e's à interroger la séparation des ordres humain et animal, et les termes spécisme et antispécisme, animalisme, véganisme, droit des animaux, ou (proto)morale animale se sont propagés dans les discours profanes, médiatiques et scientifiques.

\subsection{Un soin éthique}

15 Le syntagme droits des animaux se multiplie en effet dans les discours. Notamment en Belgique, où il est interdit d'élever des poules en cage, et en Allemagne, où l'on compte en 2017 environ 8 millions de végétarien'ne's et 1,3 million de personnes vegan. En février 2017, le ministère allemand de l'environnement décide de supprimer la viande de ses menus, tandis que l'actuel gouvernement travaille à la mise en place d'un label «bien-être animal » pour les produits carnés ${ }^{1}$.

On voit que la cause animale se présente avant tout en termes de souffrance et d'alimentation. Ce souci éthique s'est toujours exprimé :

Quiconque a entendu les cris d'un animal qu'on tue ne peut plus jamais manger de sa chair. (Confucius, 551 av. J.-C.-479 av. J.-C.)

$\mathrm{Tu}$ as défini l'homme comme le Roi des Animaux; moi par contre, je dirai que l'homme est le roi des fauves féroces parmi lesquels tu es le plus grand. N'as-tu pas effectivement tué et mangé les animaux pour satisfaire les plaisirs de ton palais, te transformant toi-même en tombe pour tous ces animaux? La nature ne produit-elle pas de la nourriture végétale en quantité suffisante pour te rassasier? (Vinci, dans Collignon $2018: 87$ )

$\mathrm{Au} \mathrm{XV}^{\mathrm{e}}$ siècle, Léonard de Vinci, végétarien de la première heure, écrit encore : « Le jour viendra où les personnes comme moi regarderont le meurtre des animaux comme ils regardent aujourd'hui le meurtre des êtres humains » (cité par Karine Lou Matignon dans Cyrulnik, Fontenay et Singer $2013: 8-9$ ). 


\subsection{Une pensée animale}

17 Si la souffrance animale a toujours été entendue, l'idée que l'animal puisse disposer d'une pensée et d'un langage s'est imposée très lentement. Au Xvi siècle, Montaigne est l'un des premiers à leur accorder la réflexion, la prévoyance, le libre arbitre, le parler et le rire ${ }^{2}$. Philosophe, Montaigne reconnaît que "L'homme se préfère d'une manière injustifiée, comme n'importe quel animal le ferait, par amour de soi » (Giocanti 2016). La faculté de raison et de langage lui permet cette " auto-proclamation logocentriste » (ibid.).

$18 \mathrm{Au} \mathrm{XVIII}^{\mathrm{e}}$ siècle, Bernardin de Saint Pierre, écrivain et botaniste français, s'oppose aux idées de Descartes et de Malebranche :

Cependant quelques philosophes, entre autres Descartes et Malebranche, ont voulu rabattre la puissance animale au-dessous de la végétale. Ils ont prétendu que les animaux n'étaient que de simples machines impassibles, ce qu'il est absurde de dire même des simples végétaux, qui sont doués d'une véritable vie, puisqu'ils se propagent par des amours. Quand on objectait à Malebranche les cris douloureux du chien frappé, il les comparait aux sons d'une cloche dans la même circonstance. Pour le prouver un jour, dans la fureur de la dispute, il tua d'un coup de pied sa propre chienne qui avait des petits. (Bernardin de Saint Pierre 1820 : 148)

Dans l'une de ses dernières œuvres majeures, The Expression of the Emotions in Man and Animals, le naturaliste anglais Charles Darwin montre au $\mathrm{xIx}^{\mathrm{e}}$ siècle les points de convergence entre psychologie humaine et comportement animal (Darwin 1872), dans l'intention de « détruire l'arrogance de l'homme » (Giocanti $2016: 1$ ).

Chez la plupart de ces penseurs, l'animal est le point de référence, l'instrument de mesure de la cruauté et de l'arrogance humaine, comme l'illustre cet aphorisme, si souvent repris sur les réseaux sociaux, de Théodore Monod, scientifique naturaliste du $\mathrm{xx}^{\mathrm{e}}$ siècle, explorateur des déserts, humaniste français: «Les animaux n'ont pas, comme l'homme, l'orgueil de se croire le roi des animaux. "

21 En 1986, l'humoriste Pierre Desproges stigmatise à son tour le sentiment de supériorité de l'humain dans l'une de ses Chroniques de la haine ordinaire qu'il consacre au règne animal: "Remarquons au passage que si l'on dit "les animaux" au pluriel, on dit "l'homme" au singulier. Parce que l'homme est unique. De même, nous dirons que les animaux font des crottes, alors que l'homme sème la merde ${ }^{3}$. "

22 À l'ironie mordante d'un Pierre Desproges s'associent des discours plus nuancés qui répondent à une philosophie utilitariste, comme ceux de l'Australien Peter Singer. Ce dernier milite contre la souffrance animale mais non contre l'idée qu'une vie peut valoir moins qu'une autre. La prise en compte du bien-être animal apparaît soit comme une fin en soi (par exemple dans le discours des animalistes et des véganistes), soit comme mode de rationalisation avec l'humain pour finalité. Matthieu Ricard, philosophe bouddhiste, rappelle dans son Plaidoyer pour les animaux que tous les êtres sont interreliés, et qu'il «ne s'agit donc pas de ne s'occuper que des animaux, mais de s'occuper aussi des animaux » (Ricard $2014: 13$ ).

\subsection{Les apports de la perspective cognitive}

Les travaux des éthologues et des primatologues (Wittig ou Andics dans Schlag 2013, Bartal et al. 2011, De Waal 2001, pour ne citer qu'eux) ont déjà largement prouvé que les 
animaux avaient des ressentis qui relevaient jadis du «propre de l'homme ». En 2018, les sciences dures se mettent de la partie, en confirmant l'existence d'une conscience animale (Le Neindre et al. 2018), mettant peut-être ainsi un terme à la mentaphobie ${ }^{4}$ qui a cours dans certains milieux éthologiques (Griffin 1992, Chauvet 2008 et 2014). Pierre Le Neindre et ses collaborateurtrice's publient en effet les résultats d'une expertise scientifique collective (ESCO) réalisée par l'Inra à la demande de l'Autorité européenne de sécurité alimentaire. Les auteur·e's, chercheur·e's des diverses disciplines mobilisées, ont analysé les données scientifiques et philosophiques publiées portant sur les capacités émotionnelles, cognitives et métacognitives des animaux, ainsi que sur les structures cérébrales et les réseaux neuronaux associés.

Les chercheur'e's en neurosciences cognitives se sont aussi penchée's sur la question du langage animal. Dans son ouvrage From hand to mouth. The origins of language, Michael Corballis (2002) consacre un chapitre à ce thème («Do animals have language?») et élabore l'hypothèse de l'origine gestuelle de la communication orale.

Des travaux en sciences cognitives ont détecté des dispositifs cognitifs chez les animaux, c'est-à-dire l'existence de représentations internes permettant d'élaborer une causalité interne et donc une capacité d'action hors d'un stimulus et d'un contexte extérieurs ; autrement dit l'existence de ce qui ressemble à une pensée autonome. Les travaux pionniers de Premack et Premack (1997) ont mis au jour des comportements de tromperie chez les primates, qui possèdent une forme de théorie de l'esprit (on a pu établir par exemple qu'ils savaient ce que les autres savent, phénomène corroboré par des études ultérieures et notamment un récent travail des chercheurs de l'Institut Max Planck (Belger et Bräuer 2018) qui ont révélé que les chiens savaient qu'ils ne savaient pas...); ceux de Frans de Waal (1996) ont montré que des orangs-outans pouvaient orner leurs têtes de végétaux, que des femelles chimpanzées s'enroulaient des plantes grimpantes autour du cou, qu'un mâle pouvait cacher une érection devant un autre mâle; de nombreuses expériences ont prouvé que les corbeaux sont remarquablement habiles dans la résolution de problèmes et qu'ils savent transférer un savoir initial à un nouveau contexte comme le montre Valérie Dufour ; les travaux de Joëlle Proust (2000), portent quant à eux sur l'existence d'états mentaux, de désirs, de croyances, voire d'intentions chez les animaux :

Les animaux non humains sont capables de catégoriser le monde en fonction d'objets et d'événements pertinents pour leur survie, et de stocker en mémoire un grand nombre d'épisodes de leur expérience passée. Ils peuvent ainsi prédire dans une certaine mesure les états du monde, et construire une carte mentale de leur territoire pour orienter leur recherche de nourriture de manière informée. Les mammifères, les serpents et les oiseaux peuvent former des représentations sur la base de la perception d'objets et d'événements distincts. Les grands primates se représentent le monde physique d'une manière voisine de celle d'un sujet humain qui n'aurait pas reçu de formation scientifique, ni n'aurait hérité de son groupe une théorie dite naïve. (Proust 2000 : § 45).

Mais en linguistique, courant $\mathrm{xx}^{\mathrm{e}}$ siècle, rares sont les linguistes qui intègrent l'animal à leur objet d'étude, si l'on excepte Erving Goffman dont l'ouvrage Façons de parler ([1981] 1987) offre de nombreuses mentions de l'animal (par exemple p. 94 à propos de l'interaction, ou p. 122 à propos des exclamations). Cela s'explique par la démarche environnementale du linguiste sociologue qui prend en considération toutes les situations, y compris celles où le'la locuteur.trice parle aux animaux et à son approche naturaliste (Pasquier 2003), l'humain étant un «animal social»: «Je crois qu'il nous 
appartient d'étudier la vie sociale des hommes comme des naturalistes, sub specie aeternatis ", affirme-t-il en effet (1987, p. 229, cité dans Pasquier 2003 : § 7).

Tous ces jalons historiques auraient pu amener bien plus tôt la question d'un langage animal dans les sciences du langage. Elle est pourtant posée dans de nombreuses disciplines, mais pas par les linguistes.

\section{Les résistances de la linguistique}

L'humaine se pense seul dans la nature, et ne sait pas toujours que « le monde sauvage est un monde de signes, d'émissions de signes qui nous interpellent, nous tiennent en éveil et avec lequel nous pouvons interagir» (Chanvallon 2016:59). Le règne de la signification ayant été pensé de manière entièrement humanocentrée, que ce soit par la philosophie, la réflexion sur la langue ou sur les signes, l'absence de l'humain est souvent synonyme d'absence de sens :

Aller dans une nature non civilisée et rencontrer la vie sauvage, c'est être seul, mais cette solitude n'est qu'une transition entre le monde humain et le monde animal. Interroger la vie sauvage, réfléchir, explorer ce monde, c'est prolonger intellectuellement et psychiquement cette aventure, c'est accepter de marcher sans repères. (Chanvallon $2016: 60$ )

On peut essayer de rassembler les arguments exploités par la linguistique ou produits par elle pour une spécificité humaine du langage et par conséquent contre l'idée d'un langage animal et plus largement non humain.

\subsection{Le frein de la théorie de l'esprit}

La prévalence du mécanisme de Descartes, de la théorie de l'instinct ou encore de l'idée pavlovienne du réflexe conditionné freinent la recherche éthologique tout autant que la recherche linguistique. La prise en compte d'une théorie de l'esprit animal passe par le prisme de certains « animaux modèles » tels les primates (Premack et Woodruff 1978) ou les dauphins. L'ethnographe Véronique Servais souligne à quel point le dauphin représente chez les Occidentaux « un état d'animalité qui, exceptionnellement, n'est pas synonyme de régression mais de progression» (Servais 2000:56). S'il est usuel d'attribuer de l'« esprit » au dauphin, cela l'est beaucoup moins pour la fourmi, l'âne ou le cochon. Le maintien d'une hiérarchie dans le règne animal se fonde sur la comparaison avec l'homme. Véronique Servais montre ainsi que l'attribution d'un «esprit » au dauphin dépend du degré de parenté reconnu entre les hommes et les animaux, et de la capacité des humainee's à se laisser "affecter » par les animaux. Inversement, dans un fameux numéro de la revue Terrain consacré aux animaux (Terrain 34), la philosophe Joëlle Proust (2000) s'intéresse aux différentes formes de communication et de cognition sociale chez l'animal non humain et modère l'enthousiasme des éthologues.

30 Finalement, la question est mal posée. Plutôt que de vouloir à toute force appliquer une théorie de l'esprit conçue par et pour l'humainee, il faut décentrer la question et rechercher ce qui est spécifique à l'animal. Cela évitera de croire à tort comme on l'a fait pour l'oiseau que certains animaux ne pensent pas, ainsi qu'en témoignent les expressions lexicalisées comme cervelle d'oiseau ou l'acception péjorative de mots et syntagmes comme pigeon ou oie blanche, autant de marques linguistiques d'une résistance spéciste qui fut largement entretenue par l'anatomiste darwinien du 
XIX ${ }^{\mathrm{e}}$ siècle Ludwig Edinger: celui-ci affirmait en effet que, faute de néocortex, les oiseaux n'étaient pas susceptibles d'intelligence (Emery 2017). Ces préjugés ont été démantelés par de nombreux scientifiques (par exemple Ackerman 2017, Kabadayi et Osvath 2017), notamment le Canadien Louis Lefebvre qui étudie depuis une trentaine d'années les capacités cognitives et neuronales des oiseaux, au moyen entre autres d'un test de quotient intellectuel aviaire (Audet, Ducatez et Lefebvre 2016, Sayol, Lefebvre et Sol 2016, Lefebvre 2001, 2018).

31 Faute de prendre en compte les capacités cognitives des animaux en tant que tels, les linguistes ne se sont pas non plus emparés des multiples études sur la communication interspécifique. Les langages artificiels comme le langage gestuel élaboré par Mary Lee Jensvold qui communique avec les singes via la langue des signes, et le langage acoustique imaginé par le spécialiste de la cognition des delphinidés, Louis Herman (ces deux exemples sont traités dans Servais 2000: 59) font l'objet d'une suspicion d'inauthenticité : ils ne semblent pas relever d'un discours véritable appuyé sur une communication authentique, le critère restant celui des humain'es. De même, le travail de Sue Savage-Rumbaugh avec le célèbre bonobo Kanzi, basé sur un système de reconnaissance de lexigrammes, mettant en évidence la possibilité d'une communication, reste encore l'objet de polémiques (Savage-Rumbaugh 1986) ${ }^{5}$.

Figure 2. Kanzi, bonobo mâle, élevé dans la langue, converse avec Sue Savage-Rumbaugh en 2006 en utilisant un « clavier » portable de symboles arbitraires que Kanzi associe aux mots.

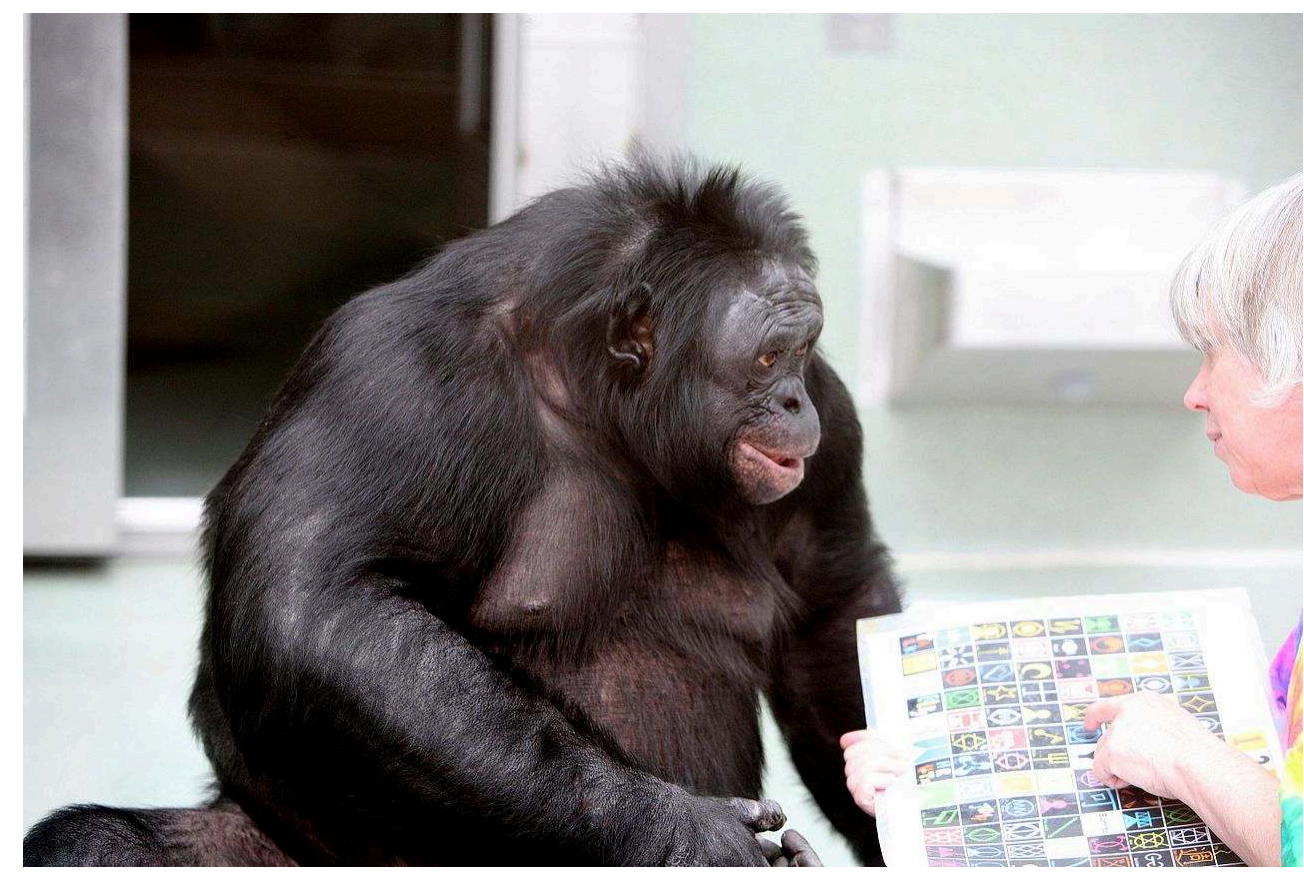

Source : Wikimedia Commons, CC-BY-SA-4.0, notre traduction.

\subsection{Les freins de la théorie linguistique}

La distinction d'avec l'animal est fondatrice de la définition du langage et de la question de son origine. Cela veut dire que la notion même de langage est d'emblée dotée du trait humain, ce qui verrouille structurellement l'accès vers d'autres types de langage. 
De manière circulaire, le déni de capacité de langage aux animaux repose sur un certain nombre de caractéristiques qui manqueraient à leurs modes de communication. On peut en mentionner six.

- Le fonctionnement symbolique du signe. La célèbre discussion entre Benveniste et von Frisch à propos de la danse des abeilles pose clairement la distinction entre le signe, que l'humaine peut produire et utiliser, et le signal, auquel l'animal est réduit (Benveniste 1952). Le signe comme le signal sont certes articulés en un signifiant et un signifié, mais le signe se combine avec d'autres pour former une chaîne syntagmatique, ce qui n'est pas le cas du signal. Aux arguments de von Frisch qui défend l'idée d'un langage des abeilles car elles possèdent différents modules de danse pour formuler des messages à leurs congénères, Benveniste répond qu'elles peuvent élaborer des signaux informatifs à partir de la réalité mais non pas combiner ces signaux pour produire du sens ; seuls les signes peuvent être combinés entre eux : «L'abeille, précise-t-il, ne construit pas de message à partir d'un autre message. [...] Or le caractère du langage est de procurer un substitut de l'expérience apte à être transmis sans fin dans le temps et l'espace, ce qui est le propre de notre symbolisme et le fondement de la tradition linguistique » (Benveniste [1952] 1966 : 61). En d'autres termes, au « signe » animal manque un signifié : il est constitué d'une forme sémiotique directement et invariablement arrimée à un référent. Il ne pourrait donc être arbitraire ou mobile, ainsi que le qualifie Bergson : «le signe instinctif [celui de l'animal] est un signe adhérent, le signe intelligent [celui de l'humain] est un signe mobile » (Bergson 1907, cité par Frei [1929] 1993 : 132).

- La double articulation du langage. Dans le langage humain, les sons dénués de sens s'articulent entre eux pour former des unités minimales douées de sens qui s'articulent à leur tour entre elles pour former des énoncés. Les systèmes de communication animaux ne fonctionnent pas de cette manière puisqu'il n'existe pas d'articulation, ni première, ni seconde : les sonssignaux sont proférés l'un après l'autre ou répétés à l'identique, même au sein de dispositifs complexes, mais non combinés. L'étude très documentée de Bouchet et al. (2016) fait cependant état de capacités combinatoires complexes chez les primates non humains. Chez les mâles mones de Campbell, par exemple, l'affixation d'une unité 'oo' élargit le sens et atténue l'urgence des cris simples d'alerte 'Krak' (signalement d'un danger au sol, le plus souvent d'un léopard) et 'Hok' (signalement de la présence d'un aigle), tandis que la composition des cris 'Boom' (incitation au rassemblement) et 'Krak-oo' informe de la chute d'un arbre.

- L'apprentissage. Une des distinctions les plus souvent avancées entre langage humain et communication animale est la possibilité de l'apprentissage. On considère que les langages ou protolangages animaux ne peuvent être appris car ils sont instinctuels et donc innés. On verra que cette impossibilité a été remise en cause par de nombreux travaux. Selon le neuroanthropologiste américain Terrence Deacon $\left(2012^{6}\right)$, le capucin en captivité est capable d'une mélodie plus complexe et plus flexible que son cousin sauvage, alors que le chant a une fonction sexuelle et que sa complexification est supposée se faire sous la pression d'une sélection sexuelle. Cette complexification inattendue (versus une stéréotypie mélodique qui serait conditionnée par des biais moteurs innés) amène Deacon à parler d'une « sélection relâchée ». Cela montre l'importance du contexte social et des préférences d'apprentissage chez les oiseaux.

- La variation. De même, les langages animaux seraient hors du champ de la variation, c'est-àdire la faculté de moduler leurs productions selon les contextes, pour les mêmes raisons. Mais plusieurs chercheurs ont montré, sur les cétacés et les oiseaux par exemple, que les animaux étaient capables de modifier leurs productions vocales, réalisant en cela des variations sociales ou comportementales. L'exemple des chauves-souris est actuellement 
parmi les plus commentés : une équipe de chercheurs israéliens a en effet montré que les vocalisations des chauves-souris n'étaient pas toutes identiques mais s'adaptaient aux interlocuteur.trice's : les jeunes chauves-souris ne communiquent pas avec leur mère de la même manière qu'avec le reste de la colonie, montrent Yosef Prat et ses collaborateur.trice's (2017). Elles sont donc capables de variation.

- La possibilité du dialogue. La communication humaine permet le dialogue entre locuteur.trice's, qui se déroule de manière imprévisible, contextuelle et créative, ce que ne permettent pas les langages animaux. Il existe des dispositifs de stimulus-réponse, mais non de dialogue avec reprise de l'énoncé de l'autre par les interlocuteur.trice's.

- La créativité et le métadiscours. Le langage humain permet la néologie, le jeu de mots, le mot d'esprit, la littérature, etc. alors que le langage animal est considéré comme régi par l'instinct ou la nécessité. Les animaux ne jouent pas avec le langage, et en ont un usage transitif, c'est-à-dire dédié à donner des informations ou agir sur le monde. Les humain·e's disposent de la possibilité de l'intransitivité du langage (au sens où l'entend Genette 1991 lorsqu'il oppose le régime de la fiction et de la poétique dont la nature est intransitive, c'està-dire sans nécessité référentielle de conformité au monde et qui suscitent une appréciation esthétique, au régime de la prose qui inversement relève du transitif). Les humain'e-s ont également la capacité de parler de leur parole, de produire du discours sur leur discours (et de faire, par exemple, de la linguistique) ; ils et elles ont accès au métadiscours.

Même si, outre l'apprentissage et la variation, on pouvait prouver que l'ensemble de ces traits sont invalidés comme spécifiquement humains, il resterait le verrou de la discipline : admettre l'existence d'un langage animal à prendre en compte comme le langage humain implique un coût théorique et épistémologique important dans la mesure où cela nécessite de remodeler considérablement la théorie du langage, ses versions mainstream et son enseignement. Ces verrouillages empêchent la linguistique de prendre en charge la réflexion sur le langage animal et entraînent un déplacement disciplinaire vers la zoosémiotique par exemple, où il est plus confortable de parler de syntaxe, de grammaire, de sémantique ou de pragmatique à propos des vocalises animales.

\section{Des catégories d'analyse pour le linguiste}

Une linguistique non spéciste, ouverte sur l'existence de langages animaux, implique de prendre acte d'une "rupture de nos encadrements conceptuels » (Calarco 2010: 85) proposée par le précurseur Uexküll (1934), puis, entre autres Derrida (2006), Lestel $(2001,2010)$ et Fontenay (1998), pour envisager les animaux dans leurs liens, leurs proximités, voire leur identité avec les humain'e-s, et non leurs distinctions ou leurs manques par rapport à ce qui serait une perfection humaine; cela en évitant la tentation anthropocentrique (voire carnophallogocentrique, selon le mot de Derrida 1989), qui est jusqu'à présent une des manières les plus fréquentes de doter l'animal d'une vie propre et d'une intelligence.

\subsection{Des sociétés animales}

Cette approche repose sur un nombre important de travaux qui, depuis une cinquantaine d'années, ont montré que les animaux faisaient société, car ils possédaient des cultures, des représentations internes, des valeurs morales et des 
facultés, si ce n'est langagières, du moins protolangagières et en toute certitude sémiotiques et communicationnelles; ce sont des appuis épistémiques importants pour le projet d'une linguistique au-delà de l'humain. On sait depuis les années 1950 que les animaux sont capables d'apprentissages et de transmissions, ce qui est caractéristique de la notion de culture: la célèbre observation par Fisher et Hinde en 1949 des mésanges ouvrant des bouteilles de lait et transmettant ce savoir au groupe en est une des premières preuves. Dès les années 1930, Jakob von Uexküll, par le célèbre exemple de la tique, avait montré que les animaux ont un monde propre du fait de leur capacité perceptive (von Uexküll 1934). L'idée de culture animale est ensuite installée en recherche par Dominique Lestel dans Les origines animales de la culture (2001) et popularisée par Frans de Waal qui a publié plusieurs ouvrages sur la question, notamment Quand les singes prennent le thé en 2001.

De multiples observations et expériences montrent également des comportements vertueux ou altruistes chez les animaux, en contexte intra- ou interspécifiques. Frans de Waal parle de leur "altruisme fonctionnel» $(1996,2010)$, des recherches en neurosciences et en anthropologie prouvent l'existence d'empathie chez les chiens, les rats ou les ouistitis. Il s'agit, comme le précise Vanessa Nurock, d'une morale "naïve " ou protomorale, qui est étroitement dépendante de l'organisation sociale :

En d'autres termes, il semble légitime de parler de «morale animale » à la condition de caractériser cette dernière comme une morale naïve - tout comme on peut affirmer que les singes sont capables d'arithmétique naïve par exemple. De sorte que la notion de « morale animale » ne peut s'entendre qu'en un sens descriptif, et non en un sens normatif permettant de faire de cette morale le critère de l'évaluation éthique de nos actions. (Nurock 2008 : § 34)

Mais il est important de préciser que la morale est liée à la capacité de faire société, et non à une capacité naturelle, un «sens moral » dont la définition ne pourrait être qu'anthropomorphique: "Il apparaît donc comme une évidence, signale Mathieu Depenau, que le phénomène moral est directement lié au phénomène social, et non au phénomène humain» (Depenau 2009: § 11). La socialité de la morale va dans le sens d'un continuum entre l'humaine et l'animal, et non d'une rupture, comme il est souvent avancé :

Contrairement à ce qu'affirmaient Thomas Huxley, collaborateur de Darwin, et les sociobiologistes de la première heure, la moralité n'émerge pas d'une rupture radicale avec les instincts naturels ni n'est l'expression d'un égoïsme génétique. Les fondements du sens moral se trouvent dans la naturalité du phénomène social, luimême constituant une des réponses les plus efficaces aux contraintes biologiques et écologiques. (Depenau 2009 : § 11)

L'existence chez les animaux d'une culture, d'une capacité cognitive et d'un sentiment moral dans le cadre d'une sociabilité vont tout à fait dans le sens d'une capacité protolangagière ou sémiotique et supportent l'hypothèse d'un langage animal.

\subsection{Des langages animaux avérés... hors de la linguistique}

Avec sa série documentaire Paroles d'animaux, diffusée les $1^{\mathrm{er}}$ et 8 décembre 2018, Arte a montré au grand public la complexité des langages animaux, depuis la fabuleuse capacité de discrimination et de mémorisation de plus d'une centaine de voix chez l'éléphant, la sophistication de la syntaxe chez certains singes ou l'élaboration du babillage des bébés chauve-souris qui apprennent à parler. Le discours animal fait son buzz, mais les linguistes continuent à se taire. Parler, voix? Les mots interdits sont 
lancés. Certes les animaux ne sont pas dotés des capacités phonatoires humaines et n'ont pas de parole "articulée » mais leur langage peut s'analyser selon des critères familiers aux linguistes et qu'étudient d'ailleurs les spécialistes non-linguistes.

À partir d'un point de vue non dualiste (vs anthropocentré) et écologique (vs logocentré), la question n'est en effet plus de savoir si les animaux parlent ou non, mais comment ils le font. On ne peut bien sûr parler spécifiquement de langage et de langue concernant la communication animale, en l'état actuel des conceptions du langage. La représentation en sciences du langage est, comme le pense Eduardo Kohn, trop étroite (le signe y est un symbole) et il faut lui préférer une conception plus large, qui y fait entrer l'icone et l'indice, pour rendre justice à la contribution animale aux discours du monde (Kohn [2013] 2017). Il devient alors possible d'envisager au moins un protolangage animal.

40 Cette idée comporte aussi une dimension politique qui nous intime d'accorder aux animaux une sentience, une voix, une subjectivité et des droits, autrement dit de les considérer comme des sujets, notamment dans la production de sens. On peut donc penser, à partir de ces orientations et de l'important corpus de recherches menées en éthologie, biologie animale, zoologie, anthropologie animale ou zoosémiotique (pour une synthèse voir Vauclair 1995), que les animaux produisent des énoncés relevant de la représentation, et des messages relevant de deux types de communication :

- les signaux intraspécifiques comportementaux (attitudes, postures, mouvements, gestes), sonores ou vocaux (cris, chants, stridulations), olfactifs (phéromones), tactiles ou constitués de modifications de l'environnement (traces, griffures, marquages de territoire); ils constituent de véritables ensembles protolangagiers (écouter par exemple les vocalisations des poules, dont Lori Marino (2017) étudie la richesse et la variété de leurs 24 vocalises), où l'on peut même repérer des dialectes, puisque des travaux sur le chant des cétacés et de certains oiseaux ont en effet révélé l'existence de variétés qui évoluent au cours du temps. 
Figure 3. Traces animales dans les vignes de Montpeyroux.

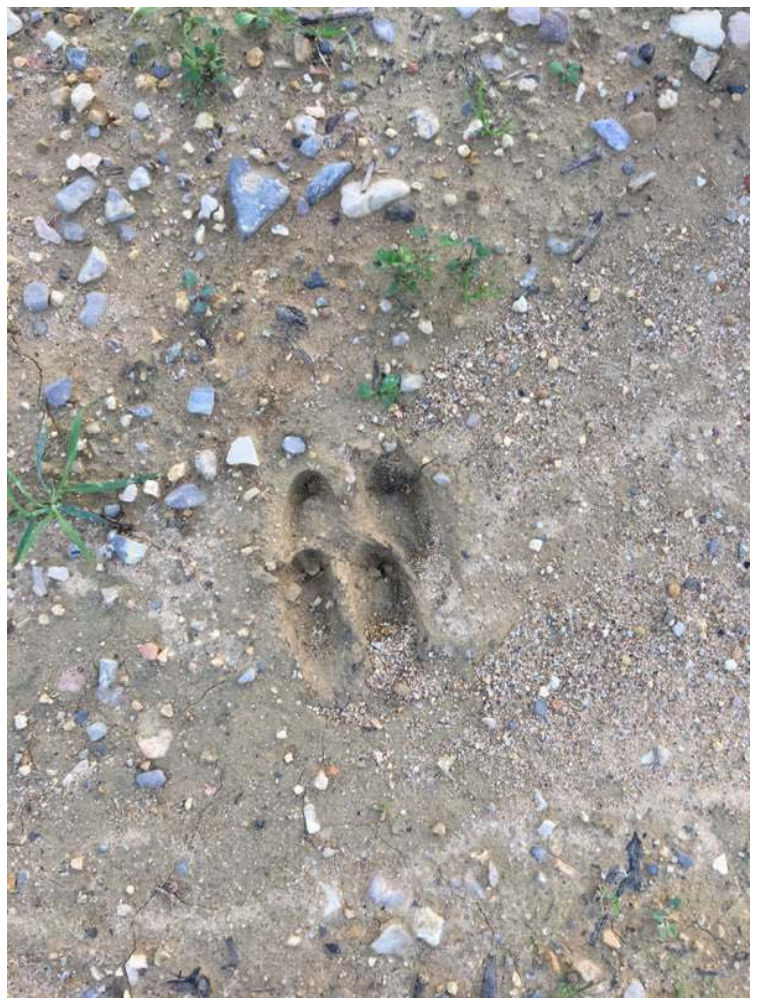

Photo Catherine Ruchon.

- les interactions interspécifiques, c'est-à-dire entre les humain'e·s et les animaux dans le cadre des « sociabilités complexes » (Baratay 2012) de ces « communautés hybrides " (Lestel 2004) : communication quotidienne entre les humaines's et leurs animaux domestiques (Mondémé 2013, 2018), dialogue musical (comme celui instauré par la compositrice Aline Pénitot avec les baleines à bosse qui répondent au son du basson et adaptent la structure de leur chant à ce son), interactions thérapeutiques entre humaine's et animaux de soin, par exemple à propos des chiens d'aveugle ou des animaux de compagnie dans le cadre de l'autisme, interactions professionnelles (compagnies cynophiles, police montée, garde républicaine, etc.) ou apprentissages du langage effectués par des animaux (Pilley et Reid 2011 sur les compétences acquises par la chienne Chaser sur les noms propres notamment). Ainsi, leur langage répond à une syntaxe. Herman (1980) affirme avoir découvert en enseignant un langage acoustique à ses dauphins que «l'arrangement syntaxique objet + verbe était plus commode et plus facilement appris que l'arrangement verbe + objet car on pouvait exiger un mouvement d'intention (une réponse d'orientation) vers l'objet avant de donner l'action à exécuter » (Herman $1980: 415$, cité par Servais $2000: 60$ ).

Le langage animal peut aussi s'analyser au travers d'une grille sémantique, comme l'ont montré Seyfarth et Cheney (2003) à propos des cris d'alarme des vervets qui transmettent à autrui de l'information à propos d'objets et d'événements du monde extérieur.

\subsection{De rares réflexions de linguistes}

Dans son récent ouvrage sur l'origine du langage (François 2018a), Jacques François fait le point sur l'état actuel de la question du langage animal en linguistique. Il explique 
que le sous-domaine de la biolinguistique ou biologie du langage, que l'on peut définir comme l'étude de la faculté de langage innée mise en place dans les années 1960 à partir des travaux de Noam Chomsky, circonscrit les facultés humaines et animales à partir du critère de la créativité :

En distinguant entre deux niveaux de la faculté de langage, étendue et étroite, les biolinguistes entendent tracer une ligne de démarcation rigoureuse entre les capacités linguistiques des espèces pratiquant des vocalisations chargées d'un sens cognitif et non seulement émotif (les cétacés et certaines espèces d'oiseaux chanteurs) dont les productions ne peuvent être que stéréotypées (relevant de la faculté de langage étendue) et celles de la seule espèce humaine, dont les capacités présentent une syntaxe créative relevant de la faculté étroite. (François 2018a : 74)

Dans leur synthèse de 2000, intitulée "The faculty of language: What is it, who has it, and how did it evolve? ", Marc Hauser, Noam Chomsky et Tecumsey Fitch développent cette conception, qui constitue la doxa sur le langage animal en linguistique actuellement (Hauser, Chomsky et Fitch 2002). « En tout état de cause, précise Jacques François, l'accord semble général sur l'importance de l'acquisition de la double aptitude à construire des représentations complexes et à les faire partager à des congénères par un discours argumenté » (François 2018a : 37). Mais il souligne cependant les progrès de l'éthologie et de la biosémiotique à partir des années 1990 et explique que la distinction entre l'homme et l'animal sur le plan des facultés langagières est devenue délicate et complexe :

On s'est ainsi aperçu que la distribution des fonctions cognitives et affectives entre les deux hémisphères du cerveau humain est partagée par la plupart des primates, et l'étude des vocalisations de certaines espèces d'oiseaux et de cétacés a révélé qu'elles peuvent présenter une grammaire et que, loin d'être limitées à des productions stéréotypées et innées, elles peuvent varier selon le contexte et faire l'objet d'un apprentissage. (François 2018a : 7)

Concernant les cétacés, il fait allusion aux travaux du biologiste Vincent Janik et de son équipe par exemple, qui a montré l'existence de dialectes chez les cétacés, c'est-à-dire de variétés dans les vocalisations (Janik 2014, Favaro, Neves, Furlati, Pessani, Martin et Janik 2016), mais également, tout récemment, l'existence de variations individuelles dans les groupes de cétacés (Sayigh et Janik 2019). Mais le primatologue Klaus Zuberbühler fait partie des chercheurees qui font avancer la linguistique sur le terrain non spéciste et non dualiste, en montrant "que les vocalisations des primates présentent un certain degré de flexibilité et qu'elles présentent des convergences entre espèces " (François 2018a: 75). Sur ce point, il y a débat entre linguistes et primatologues, qui se concentre sur l'apprentissage social, critère essentiel de « linguisticité » si l'on peut dire, pour les linguistes :

Deux interprétations sont en concurrence, (a) celle des biolinguistes qui considèrent qu'« une telle flexibilité vocale ne peut pas résulter d'un apprentissage social », et relève donc du patrimoine génétique de l'espèce, et (b) celle de Zuberbühler qui observe que les vocalisations en cause se produisent typiquement durant des interactions sociales, et qui suppose qu'elles proviennent d'un apprentissage social. (François 2018a : 75)

43 Au cas des primates et des cétacés s'ajoutent ceux des oiseaux, et l'on citera le célèbre perroquet gris du Gabon Alex (sigle pour Avian Learning EXperiment), enseigné par l'ornithologue anglaise Irène Pepperberg quasiment de sa naissance (1976) à sa mort en 2007. Alex possédait environ 150 mots d'anglais qu'il pouvait utiliser en contexte, c'està-dire au sein de conversations, et en comprenait environ un millier ${ }^{7}$. Alex a été également la première créature non humaine à poser une question. 


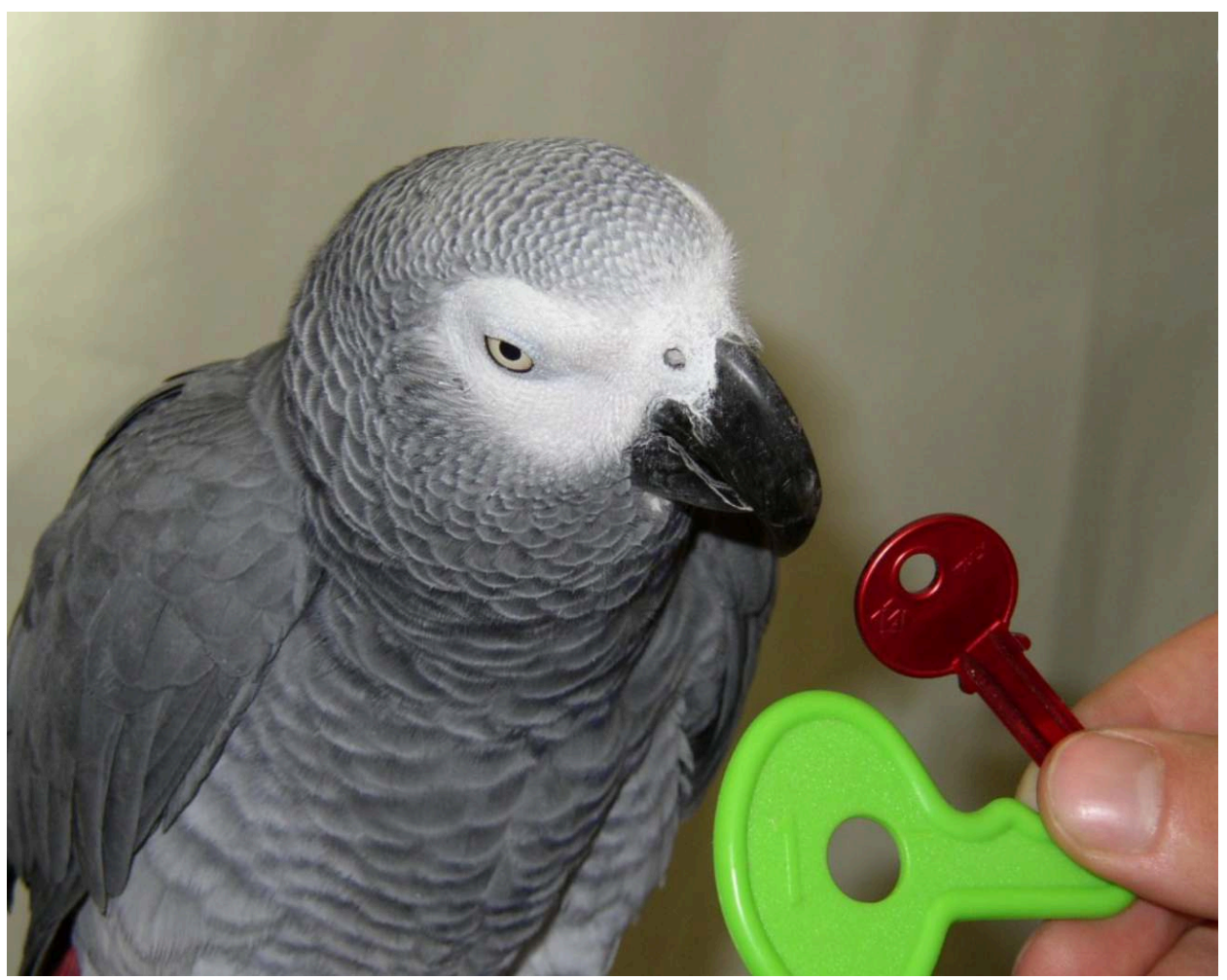

Photo extraite du site The Alex Fondation : https://alexfoundation.org/the-birds/alex/\#

Ces avancées dues aux biologistes dans les trente dernières années n'ont pas significativement ébranlé la doxa spéciste et humanocentrée des linguistes. Jacques François souligne cependant l'analogie entre la faculté de langage animale et la faculté humaine :

À défaut d'homologie, la syntaxe des vocalisations d'oiseaux chanteurs présente plusieurs analogies remarquables avec la parole humaine: une période de sensibilité pendant laquelle l'exposition au système adulte permet au développement de s'accélérer; un stade de pratique du babil où les jeunes s'entraînent avec les sons qui deviendront finalement une partie de leur répertoire ; un besoin d'apprendre non seulement les sons à produire mais aussi le contexte approprié dans lequel produire des vocalisations spécialisées; l'aptitude à traiter hiérarchiquement des séquences vocales structurées, lesquelles ont sans doute été des précurseurs de la syntaxe chez les humains; et des structures cérébrales latéralisées dédiées à l'acquisition, au stockage et à la production de vocalisations. (François 2018a: 76)

Les linguistes qui, comme Jacques François, s'intéressent au langage animal et le prennent en compte dans leurs questionnements sur la linguistique sont rares en France. Citons le GASP 8 (Groupe d'Activités Sémiotiques de Paris 8, dirigé par Denis Bertrand et Michel Costantini), qui propose un renouvellement de la zoosémiotique en travaillant sur l'énonciation ou la sémiose animale, dans une perspective cependant plutôt analogique et majoritairement littéraire (Bertrand et Costantini 2018): les chercheure's y travaillent, dans une optique sémiotique, sur « l'expression animale » et "l'énonciation animale", souvent en lien avec la littérature, mais ne font pas directement l'hypothèse d'un langage animal appuyée sur les acquis de la biologie et de l'éthologie. Ils s'arrêtent, si l'on peut dire, aux portes de l'énonciation entendue comme 
production de signes, comme le montre le travail de Nicole Pignier qui attribue aux animaux "une capacité à énoncer, c'est-à-dire à produire un ensemble de signes donnés à interpréter à autrui, avec une intentionnalité particulière » (Pignier 2018, en ligne). La zoopoétique que le groupe a mise en place ne poursuit donc pas les objectifs de description d'un langage animal qui modifierait les fondements épistémologiques de la linguistique. Seule semble-t-il actuellement Chloé Mondémé, qui publie un article dans le présent numéro, prend à bras-le-corps l'idée d'un langage animal par le biais multimodal et conversationnaliste. Elle envisage en effet un véritable échange conversationnel entre l'humain et l'animal, analysé au moyen des outils de la linguistique interactionnelle (Mondémé 2013, 2018 et ici même).

\subsection{L'exemple de la chienne Chaser}

Nous voudrions pour finir présenter le travail d'une équipe comprenant un sociologue et trois linguistes sur la chienne Chaser, célèbre border collie née en 2004, entraînée par son maître, le psychologue John W. Pilley, à mémoriser des noms d'objet.

Figure 5. Chaser et John Pilley, 2014.

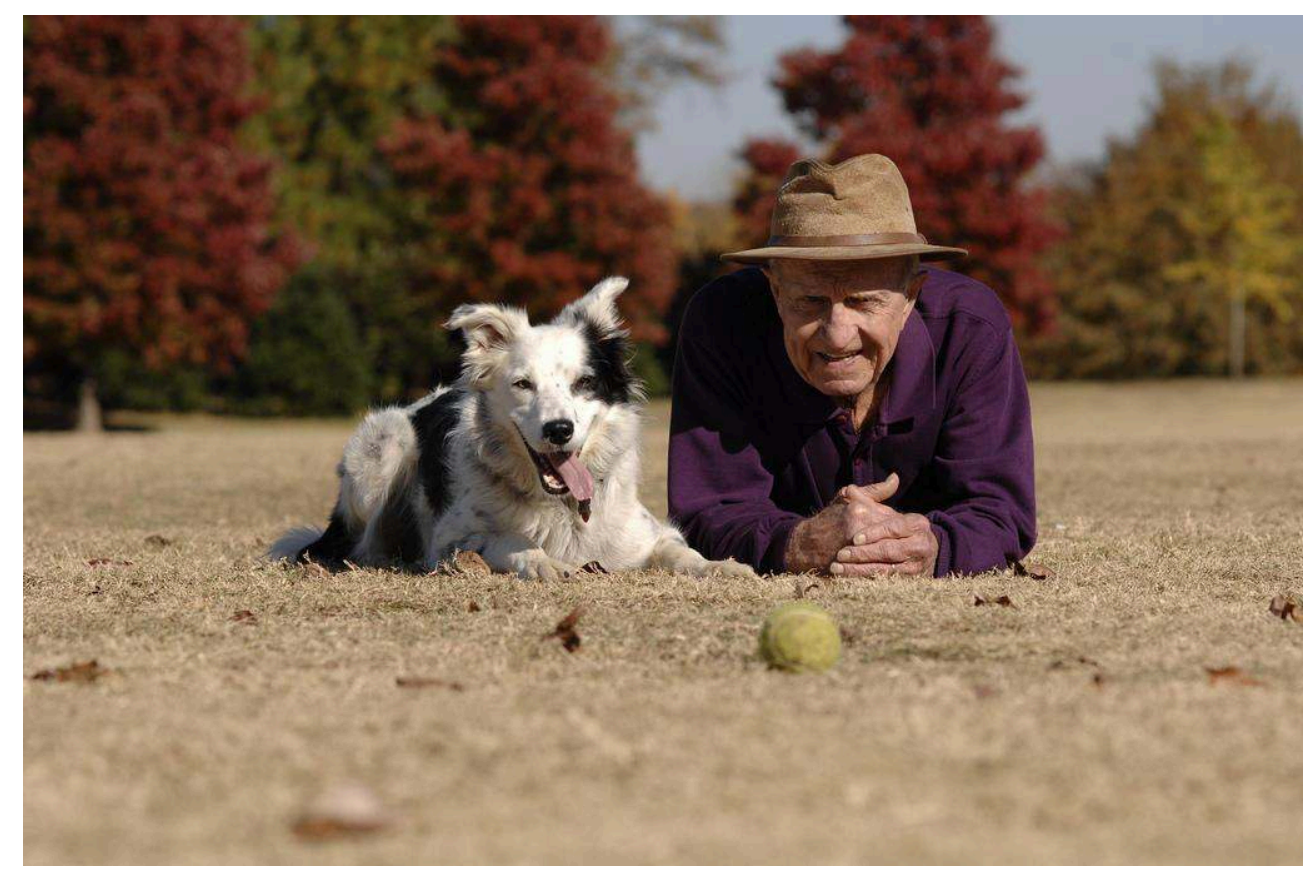

Photo extraite du site Chaser the Border Collie : https://www.chaserthebc.com/

Dans un article intitulé «Anthropologie clinique et langage animal », publié dans une revue inhabituelle pour des linguistes, Études rurales, Jean-Michel Le Bot, Clément de Guibert, Laurence Beaud et Patrice Gaborieau analysent le travail de John Pilley et Alliston Reid qui ont réalisé quatre expériences avec Chaser, pour démontrer sa capacité à apprendre des "noms propres"; à distinguer les "noms propres" des objets, d'une part, et les termes de commandement (verbes), d'autre part ; à apprendre des «noms communs"; et enfin à apprendre des noms d'objets "par exclusion». Le travail accompli avec Chaser concerne trois points particuliers, la nomenclature, le discernement perceptif et la mémorisation. L'équipe française en tire une conclusion intéressante sur les différences entre les capacités de Chaser et celle d'un être humain : 
Il y a bel et bien des similitudes entre ce que réalise Chaser et ce que réalise un être humain. Chaser est incontestablement capable de discriminer visuellement des objets entre eux sur la base d'indices perceptifs. Elle est capable de discriminer entre elles les séquences sonores qui correspondent aux différents items ou «noms» qui lui ont été appris. Elle est capable d'associer de façon bijective une série importante d'items à une série importante d'objets, et de mémoriser durablement ces associations. Elle est également capable de discriminer et de délimiter deux items inclus dans une seule séquence sonore et de comprendre spontanément leur combinaison. Mais ces similitudes ne suffisent cependant pas à parler d'identité entre ce que réalise chaser et ce que réalise un enfant, contrairement à ce qu'affirment trop rapidement John Pilley et Alliston Reid [2011]. (Le Bot et al. 2012 : § 42)

Ces réserves ne constituent cependant pas une négation des capacités langagières de la chienne, au contraire :

[...] si l'on peut reprocher parfois aux sciences humaines, non sans raisons, d'ignorer superbement les travaux des sciences de la vie, l'inverse n'est pas moins vrai. Il en résulte finalement une sorte d'anthropomorphisme scientifique préjudiciable à la connaissance de l'animal : à trop vouloir affirmer, en dépit de différences bien réelles, que les capacités et les performances de l'animal sont identiques à celles de l'homme, on risque de ne pas prêter suffisamment attention à la spécificité de ces performances et à ne pas rendre à l'animal ce qui lui est dû. (Le Bot et al. 2012 : § 47)

On a donc là une critique de l'anthropomorphisme, qui permet par un paradoxe qui n'est qu'apparent, d'ouvrir des pistes pour une meilleure compréhension du fonctionnement langagier animal :

Ainsi, dans le cas présent, l'expérience confirme l'absence de structuration de type grammatical chez l'animal, mais elle invite dans le même temps à mieux explorer le processus de perception, en apportant sur ce point des arguments supplémentaires en faveur d'une identité entre le fonctionnement animal et le fonctionnement humain. (Le Bot et al. 2012 : § 48) dans l'analyse du langage animal: si l'anthropomorphisme peut parfois fonctionner comme un "starter » des investigations du langage animal (car il permet d'écarter l'anthropodéni et de s'autoriser à penser des capacités animales analogues à celles de l'humain), il peut également constituer un puissant verrou, à l'instar de ceux décrits plus haut. Cela équivaut, dans une formulation qui sonnera peut-être de manière contre-intuitive, à réduire l'animal à l'humain; une posture plus efficace, et plus scientifique par ailleurs, est de considérer l'animal en soi, de son point de vue, en tentant de résoudre les problèmes d'accès à ses facultés perceptives, cognitives et langagières.

\section{Conclusion}

Les animal studies ${ }^{8}$ se développent, occupant désormais les champs disciplinaires de la philosophie, des études littéraires, de l'histoire, du droit, des sciences naturelles et des sciences sociales comme la sociologie ou l'anthropologie. Les études attestent des propriétés sophistiquées du langage animal (notamment de variations vocaliques sociales) et des compétences d'apprentissage (par exemple discrimination d'objets et de sons, distinction de noms propres et de noms d'objets). Les linguistes accompagnent très tardivement ce «tournant animal » (Delon 2015), se préoccupant essentiellement des aspects lexicaux (par exemple Catherine Kerbrat-Orecchioni (2018) sur l'emploi 
problématique du mot animal en tant qu'auto-hyperonyme) ou des aspects stylistiques dans les textes littéraires (Milcent-Lawson 2017 sur la zoopoétique). Le questionnement sur un langage animal intra- et interspécifique dépasse largement ces considérations. L'étude de la cognition animale et de la linguisticité des productions animales, mais sûrement aussi de la communication végétale, sont probablement le cœur même de la linguistique. De la question animale à la cause animale, de la tradition utilitariste au posthumanisme et à l'antispécisme, l'animal est aujourd'hui devenu un sujet que ne peuvent plus contourner les linguistes. Demain peut-être ce sera au tour des plantes de venir chahuter l'ordre établi dans les théories du sujet.

\section{BIBLIOGRAPHIE}

Ackerman, Jennifer, 2017, Le génie des oiseaux, Paris, Marabout.

Aristote, La Politique, [330 av. J.-C.] 1962, I, 2, trad. Jules Tricot, Paris, Vrin.

Aristote, Histoire des animaux, [vers 343 av. J.-C.] 1883, Livre I, trad. J. Barthélémy-Saint-Hilaire, Paris, Librairie Hachette et Cie.

Audet, Jean-Nicolas, Ducatez, Simon et Lefebvre, Louis, 2016, « Bajan Birds Pull Strings: Two Wild Antilean Species Enter the Select Club of String-Pullers ", PLoS ONE 11(8): e0156112, [En ligne], https://doi.org/10.1371/journal.pone.0156112, consulté le 2 juin 2019.

Baratay, Éric, 2012, Le point de vue animal. Une autre version de l'histoire, Paris, Seuil.

Bartal, Ben-Ami, Decety, Jean, Mason, Peggy, 2011, « Empathy and Pro-Social Behavior in Rats ", Science, 334 (6061), p. 1427-1430, [En ligne, version auteur], https://www.ncbi.nlm.nih.gov/pmc/ articles/PMC3760221/, consulté le 3 juin 2019.

Belger, Julia et Bräuer, Juliane, 2018, « Metacognition in dogs: Do dogs know they could be wrong? ", Learning \& Behavior, n 46, p. 398-413, https://doi.org/10.3758/s13420-018-0367-5, consulté le 23 juin 2020.

Benveniste, Émile, [1952] 1966, « Communication animale et langage humain », dans Problèmes de linguistique générale 1, Paris, Gallimard, p. 56-62.

Bernardin de Saint Pierre, Jacques-Henri, 1820, Cuvres complètes de Jacques-Henri-Bernardin de Saint-Pierre: Voyage à l'île-de France, tome I, livre V, « Harmonies animales », Bruxelles, Auguste Wahlen et Compagnie, imprimeurs-libraires.

Bertrand, Denis et Costantini, Michel (dir.), 2018, La parole aux animaux. Conditions d'extension de l'énonciation, Actes de la journée d'étude de Paris 8 (27 janvier 2017) organisée par le Groupe d'Activités Sémiotiques de Paris 8 (GASP8), Fabula, [En ligne], http://www.fabula.org/colloques/ sommaire5363.php, consulté le 2 juin 2019.

Bouchet, Hélène, Coye, Camille et Lemasson, Alban, 2016, « Le langage est-il le propre de l'humain? Apports des études sur les primates non humains ", Tétralogiques, $\mathrm{n}^{\circ} 21$, [En ligne], http://www.tetralogiques.fr/spip.php?article30, consulté le 8 juin 2019. 
Calarco, Matthew, 2010, « Nul ne sait où commence ni où finit le visage. L'humanisme et la question de l'animal », trad. d'un texte inédit par H.-S. Afeissa, dans H.-S. Afeissa et J.-B. Jeangène Vilmer (dir.), Philosophie animale. Différence, responsabilité et communauté, Paris, Vrin, p. 84-124.

Chanvallon, Stéphanie, 2016, « Regard sur la rencontre animale et pistes méthodologiques », Natures Sciences Sociétés, $\mathrm{n}^{\circ}$ 24, p. 57-66.

Chauvet David, 2008, La mentaphobie (ou comment le cri de la carotte) tue les animaux, Gagny, Droit des animaux.

Chauvet David, 2014, Contre la mentaphobie, Nouvelle version augmentée, Lausanne, Éditions L’Âge d'Homme.

Collignon, Roland A. E., 2018, Léonard de Vinci, tome 2, s. 1., Lulu.com.

Corballis, Michael, 2002, From Hand to Mouth. The Origins of Language, Princeton, Princeton University Press.

Cyrulnik, Boris, Fontenay, Élisabeth (de) et Singer, Peter, 2013, Les animaux ont aussi des droits, Paris, Seuil.

Darwin, Charles, 1872, The Expression of the Emotions in Man and Animals, Londres, John Murray. Deacon, Terrence, W., 2012, «À propos de l'homme, ou comment repenser la sélection naturelle du langage humain ", Labyrinthe, $\mathrm{n}^{\circ} 38$, trad. Marc Aymes, [En ligne], https://doi.org/10.4000/ labyrinthe.4242, consulté le 23 juin 2020.

Delon, Nicolas, 2015, « Études animales : un aperçu transatlantique », Tracés, n ${ }^{15}$, p. 187-198, [En ligne], https://doi.org/10.4000/traces.6274.

Depenau, Mathieu, 2009, « L'animal moral », Le Portique, n 23-24, [En ligne], http://

journals.openedition.org/leportique/2445, consulté le 2 juin 2019.

Derrida, Jacques, 1989, " "Il faut bien manger" ou le calcul du sujet », entretien avec Jean-Luc, Nancy, Cahiers confrontation, $\mathrm{n}^{\circ} 20$, p. 91-114.

Derrida, Jacques, 2006, L'animal que donc je suis, Paris, Galilée.

Descartes, René, [1637] 2000, Le Discours de la méthode, Paris, Garnier Flammarion.

Descola, Philippe, 2006, Par-delà nature et culture, Paris, Seuil.

De Waal, Frans, [1996] 1997, Le Bon Singe : les bases naturelles de la morale, trad. Bayard/Centurion, Paris, Bayard.

De Waal, Frans, 2001, Quand les singes prennent le thé : de la culture animale, trad. Jean-Paul Mourlon, Paris, Fayard.

De Waal, Frans, 2010, L'Âge de l'empathie : leçons de nature pour une société plus apaisée ?, trad. MarieFrance de Paloméra, Paris, Les Liens qui libèrent.

De Wit, Hendrik et Cornelius, Dirk, 1994, Histoire du développement de la biologie, vol. III, Lausanne, Presses Polytechniques et Universitaires Romandes.

Digard, Jean-Pierre, 2018, L'Animalisme est un anti-humanisme, Paris, CNRS Éditions.

Digard, Jean-Pierre, 2000, compte rendu de Terrain, $\mathrm{n}^{\circ} 34$, « Les animaux pensent-ils ? », Paris, Éditions du Patrimoine, Etudes rurales, [En ligne], https://doi.org/10.4000/etudesrurales.50, consulté le 24 mai 2019.

Emery, Nathan, 2017, L'Étonnante Intelligence des oiseaux, trad. Mickaël Legrand, Versailles, Quae. 
Favaro, Livio, Neves, Silvana, Furlati, Stephano, Pessani, Daniela, Martin, Vidal et Janik, Vincent M., 2016, « Evidence suggests vocal production learning in a cross-fostered Risso's dolphin (Grampus griseus) », Animal Cognition, vol. 19, n 4, p. 847-853, https://doi.org/10.1007/ s10071-016-0961-x, consulté le 20 mai 2019.

Fisher, James et Hinde, Robert, 1949, « The opening of milk bottles by birds », British Birds, $\mathrm{n}^{\circ} 42-11$, p. $347-357$

Fontenay, Élisabeth (de), 1998, Le Silence des bêtes : la philosophie à l'épreuve de l'animalité, Paris, Fayard.

François, Jacques, 2018a, La Genèse du langage et des langues, Paris, Éditions Sciences humaines.

François, Jacques, 2018b, De la généalogie des langues à la génétique du langage. Une documentation interdisciplinaire raisonnée, Louvain, Peeters.

Frei, Henri, [1929] 1993, La grammaire des fautes, Genève-Paris, Slatkine.

Genette, Gérard, 1991, Fiction et diction, Paris, Seuil.

Giocanti, Sylvia, 2016, « Montaigne et l'animalité », ENS Lyon, Université de Toulouse II-Le Mirail/ UMR 5037 CERPHI, http://ecole-thema.ens-lyon.fr/IMG/pdf/Article_Giocanti-2.pdf, consulté le 24 mai 2019.

Goffman, Erving, [1981] 1987, Façons de parler, trad. Alain Kihm, Paris, Minuit.

Griffin, Donald, [1992] 2001, Animal Minds: Beyond Cognition to Consciousness, Chicago, University of Chicago Press.

Hauser, Marc, Chomsky, Noam et Fitch, W. Tecumsey, 2002, "The faculty of language: What is it, who has it, and how did it evolve? ", Science, $n^{\circ} 298$, p. 1569-1579.

Herman, Louis M., 1980, « Cognitive characteristics of dolphins », dans L. H. Herman (dir.), Cetacean Behavior. Mechanisms and Functions, Malabar (Flo.), Robert E. Krieger, p. 363-429.

Janik, Vincent M., 2014, « Cetacean vocal learning and communication », Current Opinion in Neurobiology, $\mathrm{n}^{\circ}$ 28, [En ligne], https://doi.org/10.1016/j.conb.2014.06.010, consulté le 24 mai 2019 , p. 60-65.

Kabadayi, Can et Osvath, Mathias, 2017, « Ravens parallel great apes in flexible planning for tooluse and bartering », Science, vol. 357, $n^{\circ} 6347$, p. 202-204.

Kerbrat-Orecchioni, Catherine, 2018, «La linguistique face à la "question animale" ", La Clé des Langues, [En ligne], Lyon, ENS de LYON/DGESCO, http://cle.ens-lyon.fr/plurilangues/langue/ miscellanees/la-linguistique-face-a-la-question-animale, consulté le 4 juin 2019.

Kohn, Fernando, [2013] 2017, Comment pensent les forêts. L'anthropologie au-delà de l'humain, trad. Grégory Delaplace, Paris, Zones sensibles.

Latour, Bruno, 1991, Nous n'avons jamais été modernes. Essai d'anthropologie symétrique, Paris, La Découverte.

Le Bot, Jean-Michel, Guibert, Clément (de), Beaud, Laurence et Gaborieau, Patrice, 2012, « Anthropologie clinique et langage animal », Études rurales, nº 189, p. 75-90.

Lefebvre, Louis, 2001, «L'intelligente cervelle des oiseaux », La Recherche, n 347, [En ligne], http://biology.mcgill.ca/faculty/lefebvre/articles/larecherche.pdf, consulté le 4 juin 2019, p. $42-45$. 
Lefebvre, Louis, 2018, « Collared doves feeding on food pellets in an urban feral cat shelter », British Birds, $\mathrm{n}^{\circ}$ 111, p. 50, [En ligne], http://biology.mcgill.ca/faculty/lefebvre/articles/ BritishBirds_2018.pdf, consulté le 3 juin 2019.

Le Neindre, Pierre, Larrère, Raphaël, Prunet, Patrick et Dunier, Muriel, 2018, La conscience des animaux, Versailles, Quae.

Lestel, Dominique, 2001, Les Origines animales de la culture, Paris, Flammarion.

Lestel, Dominique, 2004, L'Animal singulier, Paris, Seuil.

Lestel, Dominique, 2010, L'animal est l'avenir de l'homme, Paris, Fayard.

Malebranche, 1678, De la recherche de la verite. Ou l'on traitte de la nature de l'esprit de l'homme, et de l'usage qu'il en doit faire pour eviter l'erreur dans les sciences, chapitre de la vérité, Éditeur chez André Pralard.

Marino, Lori, 2017, « Thinking chickens: a review of cognition, emotion, and behavior in the domestic chicken », Animal Cognition, n 20, p. 127-147.

Maris, Virginie, 2016, Philosophie de la biodiversité : petite éthique pour une nature en péril, Paris, Buchet Chastel.

Michalon, Jérôme, 2017, « Les Animal Studies peuvent-elles nous aider à penser l'émergence des épistémès réparatrices?", Revue d'anthropologie des connaissances, vol. 11, n 3, p. 321-349, [En ligne], https://doi.org/10.3917/rac.036.0321, consulté le 10 juin 2019.

Milcent-Lawson, Sophie, 2017, «Zoographies. Traitements linguistique et stylistique du point de vue animal en régime fictionnel », Revue des sciences humaines, $\mathrm{n}^{\circ} 328$, Université de Lille, p. 91-106.

Mondémé, Chloé, 2013, Formes d'interactions sociales entre hommes et chiens. Une approche praxéologique de la relation interspécifique, thèse de doctorat, ENS de Lyon.

Mondémé, Chloé, 2018, « Comment parle-t-on aux animaux ? Formes et effets pragmatiques de l'adresse aux animaux de compagnie », Langage et société, $\mathrm{n}^{\circ}$ 163, p. 77-99.

Nurock, Vanessa, 2008, « Les animaux sont-ils des êtres humains sympathiques ? Perspectives cognitives sur la question d'une "morale animale" ", Revue du MAUSS, n 31, p. 397-410, [En ligne], https://doi.org/10.3917/rdm.031.0397, consulté le 9 juin 2019.

Pasquier, Sylvain, 2003, « Erving Goffman : de la contrainte au jeu des apparences », Revue du MAUSS, $\mathrm{n}^{\circ}$ 22, https://doi.org/10.3917/rdm.022.0388, consulté le 24 mai 2019, p. 388-406.

Pignier, Nicole, 2018, «L'énonciation animale : une praxis énonciative en lien avec le vivant?", dans B. Denis et M. Costantini (dir.), La parole aux animaux. Conditions d'extension de l'énonciation, Fabula Les colloques, [En ligne], https://www.fabula.org/colloques/document5366.php, consulté le 24 mai 2019.

Pilley, John W. et Reid, Alliston K., 2011, « Border collie comprehends object names as verbal referents ", Behavioural Processes, n 86-2, p. 184-195.

Platon, 2016, Théétète, trad. Michel Narcy, Paris, Flammarion.

Prat, Yosef, Azoulay, Lindsay, Dor, Roi et Yovel, Yossi, 2017, « Crowd vocal learning induces vocal dialects in bats: Playback of conspecifics shapes fundamental frequency usage by pups ", PLOS Biology, [En ligne], https://doi.org/10.1371/journal.pbio.2002556, consulté le 4 juin 2019.

Premack, David et Woodruff, Guy, 1978, « Does the chimpanzee have a theory of mind? ", Behavioral and Brain Sciences, $\mathrm{n}^{\circ}$ 1, p. 515-526. 
Premack, David et Premack, Ann, 1997, «Infants attribute value +/- to the goal-directed actions of self-propelled objects ", Journal of Cognitive Neuroscience, $n^{\circ}$ 9, p. 848-856.

Proust, Joëlle, 2000, « L'animal intentionnel », Terrain, n 34, p. 23-36, [En ligne], https://doi.org/ 10.4000/terrain.944, consulté le 24 mai 2019.

Ricard, Matthieu, 2014, Plaidoyer pour les animaux. Vers une bienveillance pour tous, Paris, Allary Éditeur.

Rondal, Jean Adolphe, 2000, Le Langage : de l'animal aux origines du langage humain, Sprimont, Mardaga.

Savage-Rumbaugh, Sue, 1986, Ape Language: From Conditioned Response to Symbol, New York, Columbia University Press.

Sayigh, Laela S. et Janik, Vincent M., 2019, « Individual Signatures in Animal Groups: Cetaceans », dans J. C. Choe (dir.), Encyclopedia of Animal Behavior, $2^{\mathrm{e}}$ éd. Oxford, vol. 1, p. 539-549.

Sayol, Ferran, Lefebvre, Louis et Sol, Daniel, 2016, « Relative Brain Size and Its Relation with Associative Pallium in Birds », Brain, Behavior and Evolution, ${ }^{\circ}$ 87, [En ligne], https://doi.org/ 10.1159/000444670, consulté le 24 mai 2019.

Schaeffer, Jean-Marie, 2007, La Fin de l'exception humaine, Paris, Gallimard.

Schlag, Gabi, 2013, Les animaux pensent-ils ?, [documentaire], Arte.

Schlag Gabi, 2016, Ce que ressentent les animaux, [documentaire], Arte.

Schopenhauer, Arthur, [1851] 2005, Parerga \& Paralipomen, Paris, Coda.

Schopenhauer, Arthur, [1839] 1879, Le fondement de la morale, trad. Auguste Burdeau, numérisé par Guy Heff, https://www.schopenhauer.fr/oeuvres/fichier/le-fondement-de-la-morale.pdf, consulté le 24 mai 2019.

Servais, Véronique, 2000, «Construire l'esprit du dauphin », Terrain, $\mathrm{n}^{\circ} 34$, p. 55-72, [En ligne], https://doi.org/10.4000/terrain.963.

Seyfarth, Robert et Cheney, Dorothy, 2003, « Signalers and receivers in animal communication », Annual Review of Psychology, $\mathrm{n}^{\circ}$ 54, p. 145-173.

Uexküll, Jakob von, [1934] 1965, Mondes animaux et monde humain suivi de Théorie de la signification, trad. Philippe Muller, Paris, Denoël.

Vauclair, Jacques, 1995, L'Intelligence de l'animal, Paris, Seuil.

Vidal, Michel et Simonneaux, Laurence, 2014, « Conceptions de l'animal au regard de dialectique objet/sujet », Éducation et socialisation, n 36, [En ligne], https://doi.org/10.4000/edso.1022, consulté le 24 mai 2019.

White, Lynn Townsend, 1967, « The Historical Roots of Our Ecologic Crisis », Science, New Series, vol. 155, n 3767, p. 1203-1207, [En ligne], http://www.jstor.org/stable/1720120, consulté le 20 mai 2019.

\section{NOTES}

1. http://lettres-sh.u-pec.fr/recherche/actualites/journee-d-etude-droits-desanimaux-et-mouvement-vegan-en-allemagne-851428.kjsp?RH=1227284333036. 
2. Montaigne, Essais, Livre II, chap. 12, cité dans Revue des deux mondes, 1854, tome 5, p. 338, [en ligne], https://fr.wikisource.org/wiki/Page:Revue_des_Deux_Mondes__1854_-_tome_5.djvu/346.

3. Pierre Desproges, 5 mars 1986, Chroniques de la haine ordinaire : le règne animal, http:// celerii.free.fr/desproges/animal.html.

4. La mentaphobie, notion proposée par Donald Griffin, spécialiste d'éthologie cognitive, est le fait de refuser l'idée d'une conscience animale comme élément entrant dans l'explication des comportements animaux (Griffin [1992] 2001).

5. Pour une synthèse des tentatives de communication avec les « autres » primates, voir Rondal (2000).

6. Sur ce point, consulter aussi François 2018b, chapitre 3, «Le questionnement évolutionnaire : langage humain et sélection naturelle ».

7. Rappelons qu'en linguistique posséder des mots et les comprendre n'est pas la même chose: on estime que comprendre un mot signifie ne pas être empêché de communiquer quand on le rencontre ; posséder un mot signifie connaître son sens et en avoir la capacité d'usage.

8. Pour une généalogie des animal studies, voir l'article du sociologue Jérôme Michalon (2017).

\section{RÉSUMÉS}

Alors que de nombreuses disciplines s'intéressent à la production de significations par les animaux, les sciences du langage résistent et campent sur des positions anthropocentrées qui les isolent dans les sciences humaines et sociales au niveau international. Dans cet article, nous tentons de comprendre ce qui bloque les linguistes dans la seule prise en compte du langage humain et d'identifier les verrous qui les empêchent de prendre en compte des éléments non humains, alors que les éthologues, philosophes, cognitivistes, anthropologues, sociologues ou psychologues posent la question du langage animal. Nous examinons d'abord les formes de cette résistance en linguistique, en étudiant trois discours spécifiques: une doxa professionnelle anthropocentrée, la prégnance d'une conception axiologique négative de l'anthropomorphisme et la construction scientifique de cette résistance, fondée sur les notions d'articulation du langage et de symbolisation. Nous identifions ensuite trois verrous pour expliquer cette absence de prise en compte du non-humain : le verrou idéologique de l'anthropocentrisme ou supériorité humaine ; un verrou épistémologique maintenant le logocentrisme comme cadre d'analyse pour la linguistique ; un verrou psycho-professionnel, proche de la dissonance cognitive, qui consiste à réfuter les propositions dont l'acceptation entraînerait un coût théorique et épistémologique trop important.

While many disciplines are interested in the production of meanings by animals, language sciences resist and camp on anthropocentric positions that isolate them in the humanities and social sciences at the international level. In this article, we try to understand what blocks linguists from taking into account only human language and to identify the locks that prevent them from taking into account non-human elements, whereas ethologists, philosophers, 
cognitivists, anthropologists, sociologists or psychologists ask the question of animal language. We first examine the forms of this resistance in linguistics by studying three specific discourses: an anthropocentric professional doxa, the prevalence of a negative axiological conception of anthropomorphism and the scientific construction of this resistance, based on the notions of language articulation and symbolisation. We then identify three locks to explain this lack of consideration of the non-human: the ideological lock of anthropocentrism or human superiority; an epistemological lock maintaining logocentrism as an analytical framework for linguistics; a psycho-professional lock, close to cognitive dissonance, which consists in refuting proposals whose acceptance would entail too great a theoretical and epistemological cost.

INDEX

Mots-clés : anthropocentrisme, anthropodéni, anthropomorphisme, langage animal, logocentrisme, vocalisations

Keywords : anthropocentrism, anthropodenial, anthropomorphism, animal language, logocentrism, vocalizations

\section{AUTEURS}

\section{MARIE-ANNE PAVEAU}

Université Sorbonne Paris Nord, Pléiade (ER 7338)

\section{CATHERINE RUCHON}

Université Paul-Valéry Montpellier 3, Pléiade (ER 7338) 